\title{
Lens-free Multi-Laser Spectral Light-Field Fusion Microscopy
}

Farnoud Kazemzadeh

Alexander Wong
University of Waterloo, ON, Canada

University of Waterloo, ON, Canada

\section{Abstract}

We present a device and method for performing lens-free spectral light-field fusion microscopy at sub-pixel resolutions while taking advantage of the large field-of-view capability. A collection of lasers at different wavelengths is used in pulsed mode and enables the capture of interferometric light-field encodings of a specimen placed near the detector. Numerically fusing the spectral complex light-fields obtained from the encodings produces an image of the specimen at higher resolution and signal-to-noise-ratio while suppressing various aberrations and artifacts.

\section{Introduction}

Lens-free microscopy has gained tremendous amount of traction over the past decade. This technique is particularly advantageous in application where a large field-of-view (FOV) is to be interrogated. Lens-free microscopes, using various super-resolution techniques, offer comparable imaging resolution to traditional microscopes with FOVs that are up to two orders of magnitude larger in size $[1,2]$.

Lens-free microscopes, also known as holographic microscopes, observe and capture the complete light-field which consists of intensity and phase information of the incoming wavefront of light. The recorded light-field encodings are then numerically processed, which is equivalent to a numerical lens, and an image of the target can be produced. This imaging modality was first proposed in the 1940s [3]; however, until recently, not much advancement had been seen because computational power was not sufficient for any practical applications of this microscopy technique. With the recent ubiquity of advance computing, the usability and advantages of this imaging technique is gaining interest.

Most lens-free microsocopy techniques impose an inherent resolution limit of the detector pixel size, meaning if all aberrations and artifacts are suppressed and optimal signal-to-noise-ratio (SNR) is achieved, the highest resolution achievable is limited by the pixel pitch. There are techniques which circumvent the pixel pitch limitation by use of complex and expensive hardware setups that reduce the effective size of a sampling pixel thereby increasing the spatial resolution.

There are two main methods for increasing the spatial resolution in lens-free microscopy: 1) light-field encoding magnification and 2) aperture scanning. In order to magnify the light-field encoding, the sample is placed father away from the detector and closer to the light source [4]. The scanning aperture technique is used to introduce a sub-pixel shift in captured light-field encodings which in turn allows for the light-field encoding to be sampled at different locations, increasing the resolution by an order of magnitude [5].

We propose a new lens-free multi-laser spectral light-field fusion microscopy device and method for resolution improvement which reduces aberrations and artifacts while increasing SNR and resolving power. The microscopy system uses light-field encodings captured by using different laser sources without the need of any elaborate scanning apparatus or complex optical setup.

\section{Methodology}

The instrument, Figure 1, consists of three lasers with wavelengths of 450,532 , and $653 \mathrm{~nm}$. The lasers are coupled to a singlemode fiber optic cable which then delivers the light to the imaging back-end. The sample, the USAF 1951 resolution target, is placed directly on the detector active area at a distance of $\sim 2 \mathrm{~mm}$. A

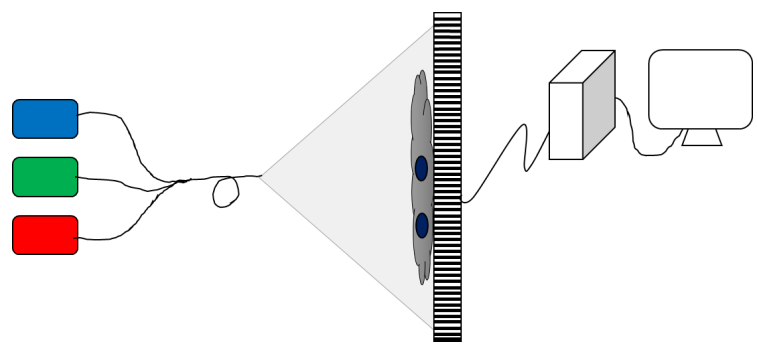

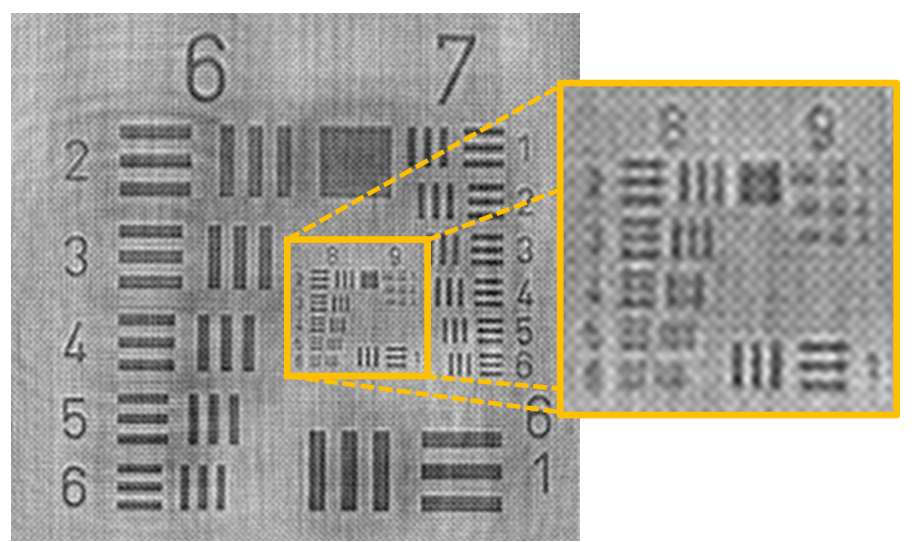

Fig. 2: The high-resolution portion of the standard USAF 1951 resolution target acquired using the proposed system. This is a portion of the $30 \mathrm{~mm}^{2}$, the distance between the lines of 8-5 is $1.23 \mu \mathrm{m}$.

monochromatic detector is used to sequentially capture the lightfield encodings at each wavelength with exposure time $<1 \mathrm{~ms}$ at each wavelength. The light-field encodings are then processed using a Bayesian-based spectral fusion technique represented by [1]

$$
\begin{gathered}
\hat{f}_{\oplus}=\int_{\lambda \in \Lambda} w_{\lambda} \operatorname{argmax}_{f_{\lambda}} p\left(g_{\lambda} \mid f_{\lambda}\right) p\left(f_{\lambda}\right), \\
p\left(g_{\lambda} \mid f_{\lambda}\right)=\prod_{s \in S} \frac{\left(\mathfrak{F}^{-1}\left\{\frac{H_{a, \lambda}}{H_{d, z, \lambda}} \mathfrak{F}\left\{f_{\lambda, s}\right\}\right\}\right)^{g_{\lambda, s}} e^{-\left(\mathfrak{F}^{-1}\left\{\frac{H_{a, \lambda}}{H_{d, z, \lambda}} \mathfrak{F}\left\{f_{\lambda, s}\right\}\right\}\right)}}{g_{\lambda, s} !} .
\end{gathered}
$$

\section{Discussion}

The imaging result from the proposed multi-laser spectral lightfusion microscopy is shown in Figure 2. The zoomed-in region shows the performance capability of the system which can successfully resolve element 5 of group 8 with separation of $1.23 \mu \mathrm{m}$. This is a $30 \%$ increase in resolution over the one prescribed by the pixel pitch of $1.67 \mu \mathrm{m}$. The numerical aperture, which describes the resolution capabilities of a microscope objective, is determined to be 0.23 with FOV $\sim 30 \mathrm{~mm}^{2}$. The proposed method produces results that are comparable to other holographic microscopes without the need of any complex optical setup. Furthermore, this microscopy modality can produce results comparable to the widelyused light microscope at the fraction of the cost.

\section{Acknowledgments}

This work was supported by the Natural Sciences and Engineering Research Council of Canada, Canada Research Chairs Program, and the Ontario Ministry of Research and Innovation.

\section{References}

[1] Kazemzadeh, F., Jin, C., Molladavoodi, S., Mei, Y., Emelko, M.B., Gorbet, M.B. and Wong, A. Lens-free spectral lightfield fusion microscopy for contrast- and resolution-enhanced imaging of biological specimens, Opt. Lett. 40(16), 3862-3865 (2015).

[2] Wong, A., Kazemzadeh, F., Jin, C., and Wang, X.Y. Bayesianbased aberration correction and numerical diffraction for improved lensfree on-chip microscopy of biological specimens, (Opt. Lett.) 40(10), 2233-2236 (2015).

[3] Gabor, D., A New Microscope Principle, Nature 16, 777-778 (1948).

[4] Noom, D.W.E., Eikema, K.S.E. and Witte, S. Lensless phase contrast microscopy based on multiwavelength Fresnel diffraction, Opt. Lett. 39(2) 193-196 (2014).

[5] Isikman, S.O., Greenbaum, A., Luo, W., Coskun, A.F., Ozcan, A. Giga-Pixel Lensfree Holographic Microscopy and Tomography Using Color Image Sensors, PloS ONE 7(9), e45044 (2012). 